\title{
Performance comparison of IEC 61850-90-5 and IEEE C37.118.2 based wide area PMU communication networks
}

\author{
Ikbal ALI $^{1}$, Mohd Asim AFTAB ${ }^{1}$, S. M. Suhail HUSSAIN ${ }^{1}$
}

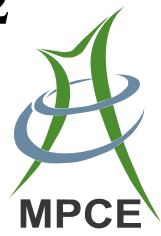

\begin{abstract}
Dynamic operation and control of power system is being increasingly done with the help of phasor measurement unit (PMU) based wide area monitoring and control system. The data communication requirements for the PMU based applications are well addressed by IEEE C37.118.2 standard. However, IEC 61850 is now becoming a leading standard for power utility automation needs. A new part of IEC 61850, IEC 61850-90-5, specifying the IEEE C37.118.1 based synchrophasors data transfer according to IEC 61850, will lead to IEC 61850-90-5 based PMU communication networks. A novel IEC 61850-90-5 compliant communication model of PMU is proposed in this paper. We also present a comparative performance analysis of the PMU communication networks supporting the new synchrophasor standard for data transfer, i.e. IEC 61850-90-5, and the existing standard, i.e. IEEE C37.118.2, for end to end (ETE) delay requirements for a modified IEEE 30 bus test system.
\end{abstract}

Keywords Phasor measurement unit, IEC 61850-90-5, IEEE C37.118.2, End-to-end delay

CrossCheck date: 24 March 2016

Received: 30 November 2015/Accepted: 1 April 2016/Published online: 9 July 2016

(C) The Author(s) 2016. This article is published with open access at Springerlink.com

$\triangle$ S. M. Suhail HUSSAIN

s.suhail.md@gmail.com

Ikbal ALI

iali1@jmi.ac.in

Mohd Asim AFTAB

mohdasimaftab4@gmail.com

1 Department of Electrical Engineering, Faculty of Engineering and Technology, Jamia Millia Islamia (A Central University), Jamia Nagar, New Delhi 110025, India

\section{Introduction}

The rapid increase in occurrence of line faults, failure of generation system and uncontrolled blackouts have led to a situation where the electrical utility needs dynamic and real time monitoring of critical parameters for power systems such as voltage, current, frequency, load angle etc. This has resulted into a prospect in control and monitoring advances employing phasor measuring unit (PMU) [1]. A PMU provides synchronized measurements of power system parameters along with a time stamp from a global positioning system (GPS) clock. PMU cannot be placed on every bus of the power system in a wide area monitoring system since placing PMUs all over the power system buses would attract huge costs and it would be very difficult to process the gathered data, because of huge processing capacity and time [2]. Thus optimal placement of PMU is resorted and determination of appropriate locations for minimum numbers of PMUs becomes a significant concern $[3,4]$.

Synchrophasor measurements are widely used for various monitoring, assessment and control applications such as state estimation [5], control of frequency fluctuation [6], torque angle control of rotor [7] etc. For such applications the synchrophasor data, measured by PMU typically at a substation in power system, is sent to the phasor data concentrator (PDC), located in a control center, through a communication network. This data transfer is to be achieved within the specified time limit so as to avoid misinterpretation and incorrect evaluation of measurements. The major portion of delay in transmission of synchrophasor data mainly depends on the communication network and underlying protocols used. Thus the communication network plays a very significant role in PMU wide area monitoring and control applications. 
The measurement and communication needs for the PMU were first addressed by IEEE 1344 synchrophasor standard which were introduced in the year 1995 [8]. In 2005, IEEE C37.118 [9] standard was published which became the foundation for synchrophasor data measurements. Later in the year 2011, this standard was split in two parts; IEEE C37.118.1 [10, 11] which addressed measurement related requirements and IEEE C37.118.2 [12] addressed the data transfer requirements of PMU. Although, the performance analysis of PMU communication networks based on IEEE C37.118.2 has been extensively reported in [13-15]. However, IEC 61850 [16] has emerged as a de-facto standard for substation automation and is also being accepted as a standard for utility automation. Since IEC 61850-90-5 [17], published in 2012, provide guidelines for IEEE C37.118.1 based synchrophasors data transfer as per IEC 61850.

Ahmad et al. [18] proposed a technique for phasor estimation using IEC 61850-9-2 process bus sample values. However, their technique did not incorporate information about routing of sample values through an IP based wide area network. Reference [19] presented an IEC 61850 based PMU, but did not provide any information on modeling of logical nodes and security aspects as per IEC 61850-90-5, which is making the first effort to demonstrate the underlying process for transmitting the IEEE C37.118.1 synchrophasor information according to IEC 61850, and to provide an information model for IEC $61850-90-5$ based PMU. Moreover, a comparative analysis of PMU communication networks having synchrophasors data transfer based on IEC 61850-90-5 and IEEE C37.118.2 is also presented in order to provide insight about the working of these two standards.

Remainder of the paper is organized as follows, the analysis of the IEEE C37.118.2 based PMU communication is presented in Sect. 2. IEC 61850-90-5 based modeling of PMU and PMU communication network is described in Sect. 3. Section 4 presents the comparative performance analysis of IEEE C37.118.2 and IEC 61850-90-5 PMU communication networks. Finally in Sect. 5, conclusion of the paper is presented.

\section{IEEE C37.118.2 based PMU communication}

A PMU is a device which provides accurate time stamping of power system information by performing synchrophasor measurements by incorporating GPS time signal for time reference. It transmits synchrophasor data to remote peers either by unicast or multicast. The remote peer is a PDC located at a control and monitoring center. Figure 1 shows the block diagram of a PMU. The power systems measurements, i.e. voltage and current phasors, are

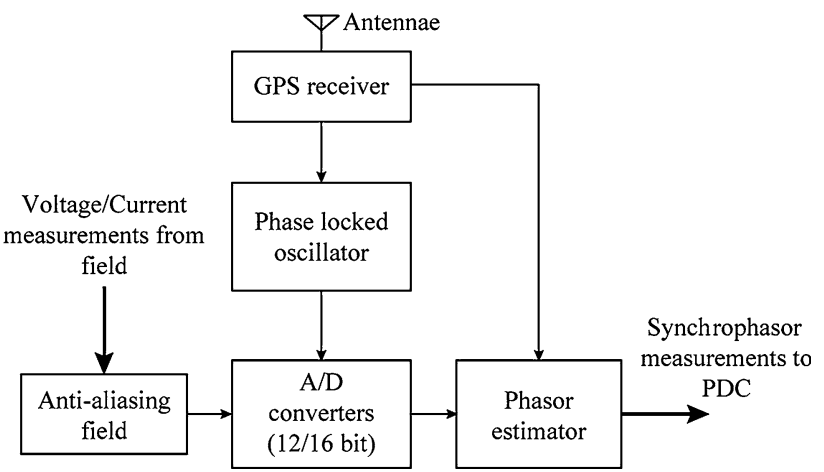

Fig. 1 Block diagram of phasor measurement unit

acquired through CTs/PTs located in field and are passed through an anti-aliasing filter so as to restrict the signal bandwidth according to sampling theorem. The input is then converted into digital format in accordance with IEEE C37.118 standard specifications which is finally sent to the phasor estimation unit. In GPS clock, a crystal oscillator is used to supply clock pulses. The phase locked oscillator corrects for any error between pulses per second and clock frequency. Finally, the time stamped phasor measurement is sent to the PDC via a communication interface.

The IEEE C37.118 standard defines methods for evaluation of synchrophasor measurements, time synchronization, application of time-tags to the measurements and format of messages to be exchanged over the network. However, the protocol guarantees complete flexibility to opt for any mode of communication. Originally, IEEE C37.118 addressed synchrophasor measurements performance under steady state conditions only, but in 2011 IEEE C37.118 was revised and split into two parts, where IEEE C37.118.1 addressed dynamic performance requirements like frequency, and rate of change of frequency (ROCOF) measurement etc. The other part IEEE C37.118.2 addresses the issues related to synchrophasor data communication.

PMU samples the synchrophasor measurements and transmits these measurements in the form of data packet to a PDC via a wide area communication network. IEEE C37.118.2 defines four types of messages for PMU communication i.e. data, configuration, header and command. The data messages correspond to measurements made by PMU. Configuration messages contains the information about calibration factors and meta data required to properly decode the data messages which are normally in machine readable format. The data word packet consists of various layers of network protocols necessary for efficient and secure message exchange over the network.

Figure 2 shows complete description of IEEE C37.118.2 type data word with different fields and its size. The "SYNC" field is of 2 bytes and is used to provide frame 


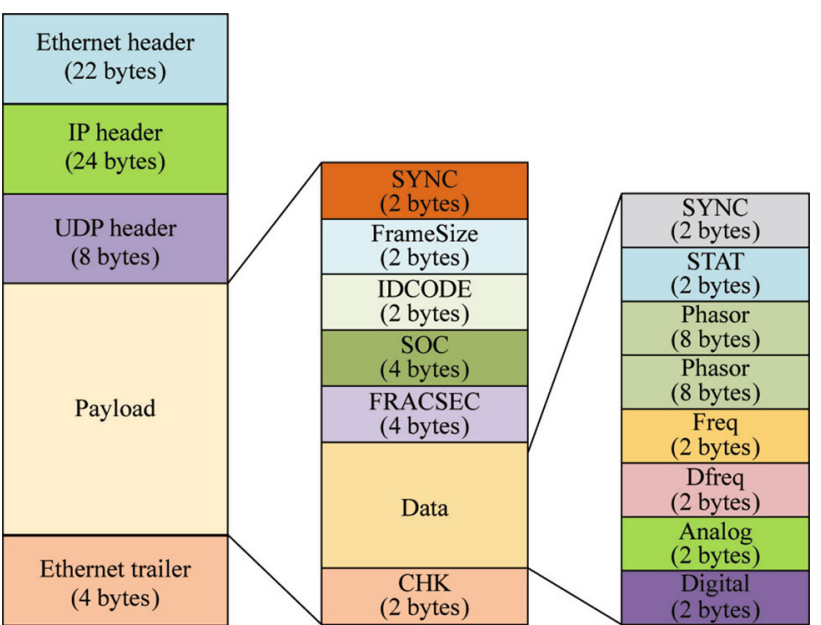

Fig. 2 Data word structure for IEEE C37.118.2 protocol

type and version number. "FRAMESIZE", "IDCODE" denotes size-of-frame and source identifier of incoming stream respectively and are of 2 bytes each. "SOC" denotes second-of-century which forms time stamp for each measurement in accordance with universal time coordinated (UTC). To provide precision to time stamp a field of 4 bytes, known as "FRACSEC" is used for denoting the fraction-of-second in the measurement. "STAT" defines the status word which is of 2 bytes and is stuffed with each block of data and provides information about the complete status of block. The "DATA" field contains various values of phasors, frequency both static and dynamic. The number of values is determined by the "PHNMR" field in configuration 1, 2, and 3 frames. There is a provision of sending analog and digital values also along with other fields in the data word. The frame is terminated by a "CHK" field of 2 bytes used for determining any error in the transmitted word based upon the technique of cyclic redundancy check. Hence the total size of a IEEE C37.118.2 data word is calculated to be 112 bytes.

Configuration messages are of three different types: CFG-1, CFG-2 and CFG-3. CFG-1 represents the reporting capability of PMU in terms of different types of data and is of 100 bytes. CFG-2 gives information about the synchrophasor measurements which are currently being transmitted/reported and it is 100 bytes. CFG-3 contains some enhanced flexibility and information about characteristics and measurements that are being sent by a PMU and its size is 110 bytes. The header messages which are 18 bytes contain the readable descriptive information sent from PMU/PDC. Command messages control operation of synchrophasor measurement devices and the transmission of data configuration and header messages and has a size of 20 bytes. For example, command messages are 'data_on', 'data_off', 'send_header' etc.
The sequence of message exchanges for transmitting synchrophasor information between PMU and PDC as per IEEE C37.118 is illustrated in the Fig. 3. PDC first sends a command message requesting PMU to send its configuration. Then the PMU responds by sending the specific configuration frame as requested by the PDC with the help of CMD field of the command word frame. The PDC after receiving the configuration of PMU now again sends a command message requesting PMU to turn on transmission of data word, i.e. synchrophasor measurements. The PMU flushes data word packets to PDC repeatedly until PDC issues turn off transmission command.

\section{IEC 61850-90-5 based PMU communication}

IEC 61850-90-5 is a part of IEC 61850 standard series which deals primarily with transmission of synchrophasor measurement information according to IEC 61850. IEC 61850 normally uses the approach of common information model (CIM) of real devices in terms of logical nodes (LN) which are capable of defining various functionalities in the implementation of real devices.

\subsection{Information modeling of IEC 61850-90-5 based PMU}

Information modelling is a method of providing standardized syntax, semantics and hierarchical structures to the data that is exchanged among different devices and systems. Logical nodes are a group of data objects (DO) which serve specific functions and have been defined conceptually in IEC 61850 standards. Combination of several logical nodes form logical device. The information contained in the attributes of logical nodes is exchanged in accordance with a set of well-established rules as per IEC 61850. Intelligent electronic devices (IEDs) are physical devices consisting of one or more logical devices. PMU is a type of IED containing the relevant LNs for synchrophasor measurements.

PMU IED is modeled by adding some new logical nodes and data objects to the existing IEC 61850 compliant merging units (MUs) which are normally used in substations. MUs sample the received power systems measurements, e.g. currents and voltages, in substations through $\mathrm{CTs} / \mathrm{PTs}$, and forward them to other protection and control IEDs. The MU IED typically contains the logical nodes for measurement information (i.e. MMXU), sequence imbalance (i.e. MSQI), current transformer (i.e. TCTR), potential transformer (i.e. TVTR) etc. The logical nodes TCTR and TVTR are used to receive the digital current and voltage values from the CT and PT respectively. The MMXU logical node contains data objects "PhV", "A", 

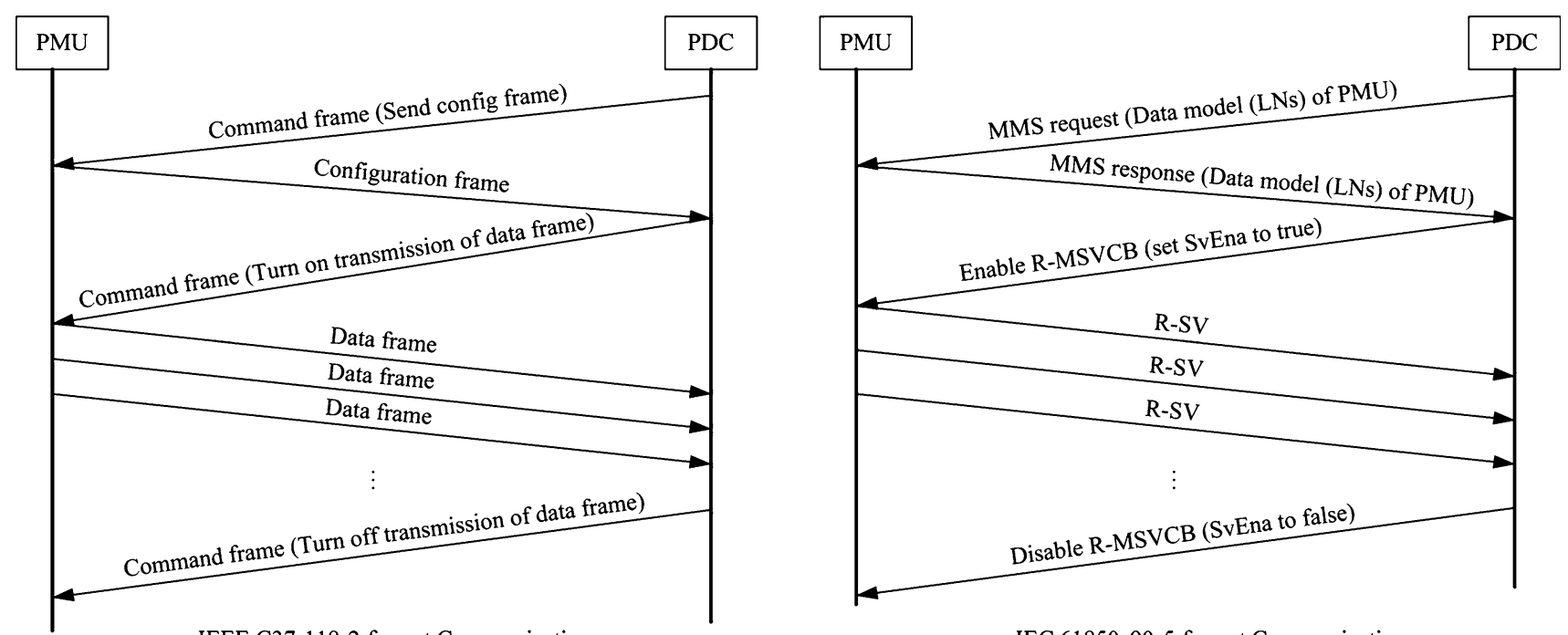

IEEE C37.118.2 format Communication

IEC 61850-90-5 format Communication

Fig. 3 Messages exchange for IEEE C37.118.2 and IEC 61850-90-5 PMUs

"Hz" which provides information about the measured data in terms of values of phase voltage, current and frequency respectively.

In order to model IEC 61850-90-5 based PMU from the existing IEC $61850 \mathrm{MU}$ IED, the requirements specified in IEEE C37.118.1 standard such as rate of change of frequency (ROCOF) must be incorporated. This additional functionality is included in IEC $61850-90-5$ by adding a new data object called "HzRte" in MMXU logical node. "HzRte" is a data object which provides the ROCOF values in floating point. Also to accomplish the sampling rates, data objects such as "ClcIntvPer", "ClcIntvTyp" and "ClcMod" are introduced in MMXU logical node. To identify the class of PMU, i.e. Protection (P) class or Measurement (M) class, a new data object "ClcMth" is also included in the MMXU logical node.

Now a novel information model of PMU in compliance with IEC 61850 is as shown in Fig. 4. The logical node LLNO addresses common issues of all logical devices and LPHD logical node provides physical information about the device such as name plate rating, control modes etc. The logical node TANG is used to provide the information

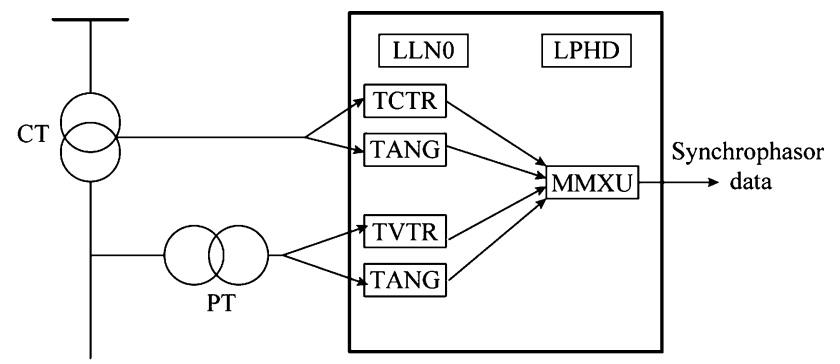

Fig. 4 Information model of IEC 61850-90-5 based PMU about angle of the measured phasor. The measurement logical node MMXU receives the samples as fast as 4000 samples/sec (for $50 \mathrm{~Hz}$ ) from the TCTR and TVTR logical nodes and provides the measured data to a PDC.

\subsection{Service modeling of IEC 61850-90-5 based PMU}

Service modeling corresponds to the exchange of different messages or services performed by a particular device or IED. As per IEEE C37.118.2 the PMU message comprises of the synchrophasors measurements, as per IEEE C37.118.1, and is sent over a WAN by mapping over UDP/IP protocols as shown in Fig. 5. IEC 61850 employs sample values (SV) and generic object oriented substation events (GOOSE) as real time communication services which are directly mapped onto the Ethernet layer and these messages could not be communicated as such over IP also shown in Fig. 5. MU IED in a SCN normally sends the
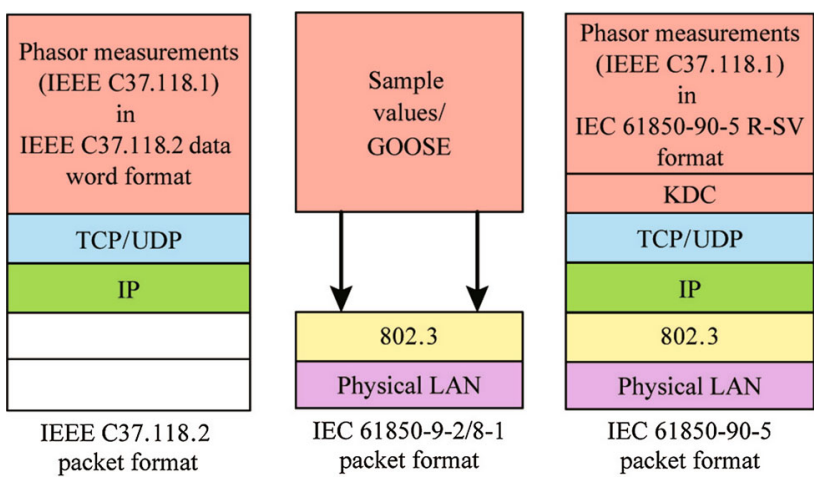

Fig. 5 Protocol stack comparison for IEEE C37.118.2 and IEC 61850-90-5 standards 
samples values by directly mapping them on to Ethernet layer of the local area network (LAN) of substations. In case of PMU, the phasor SVs has to be generally transmitted over wide area network (WAN). In order to use SV and GOOSE for transmitting PMU data over a WAN, they must be mapped onto a routable UDP/TCP service having multicast capabilities. Hence these routable SV and GOOSE messages after adding network and transport layers are termed as R-SV and R-GOOSE. Due to the periodic nature of these services, UDP with multicast addressing is normally the transport protocol chosen for this purpose. The new mapping of the SV service is based on routable UDP, and therefore in short it is called R-SV. Figure 5 also shows the protocol stack of the IEC 61850-90-5 based PMU.

IEC 61850-9-2 and 8-1 standards already have control blocks for SVs, i.e. streaming information, and GOOSE messages, i.e. event driven information, which are known as "SVCB" and "GoCB" control blocks respectively. These control blocks are used to start and stop the flow of messages. Control blocks for R-SV and R-GOOSE are defined by addition of new functional constraints to the existing control blocks. So in order to accommodate the IP protocol in SVCB and GoCB control blocks, new attributes such as "SecurityEnable" and "UDPCOMADDR" are introduced. The "UDPCOMADDR" attribute contains the priority, VID, and other attributes related to IP such as IP address, IP type of service etc. The new control blocks for R-SV and R-GOOSE, with additional enhancements, are termed as R-MSVCB and R-GoCB respectively.

The message format of IEC 61850-90-5 compliant PMU is shown in Fig. 6. The Sample Value Protocol Data Unit (SVPDU) service in the session header has been expanded in the figure. In order to accommodate MSVCB control block and time stamp of measurement, Application Service Data Unit (ASDU) is modified to accommodate two new fields "MsvID" and " $\mathrm{t}$ " in the existing IEC 61850-9-2 ASDU frame also shown in Fig. 6. The R-SV message size will be 305 bytes long for 4 ASDUs.

The service exchanges among the PMU and PDC in IEC 61850-90-5 model takes place as illustrated in Fig. 3. When, PDC sends a MMS-request type message to obtain the information model of the PMU. This service is equivalent of a command message for obtaining configuration frame in IEEE C37.118.2. Then PMU sends back a MMSresponse type message with the information of all its LNs. After receiving the information model, PDC sets "SvEna" value of R-MSVCB to true. The PMU continuously transmits R-SV values to PDC until the PDC sends command to turn off transmission by setting "SvEna" to false in R-MSVCB.

\subsection{Security features}

The security features forms an integral part of synchrophasor data communication and is left mostly unaddressed or merely addressed in IEEE C37.118 standard. The prominent characteristics employed to judge security in wide area communication systems includes confidentiality, integrity, and availability. 'Confidentiality' deals with information privacy in the data packet when it is transmitted over an insecure communication network. It provides a measure of vulnerability of the data packets to attackers and is achieved through data encryption. IEEE C37.118 does not address confidentiality issue. However in IEC 61850-90-5, confidentiality is achieved by implementing the concept of "perfect-forward" security and encryption key rotation between publishers and

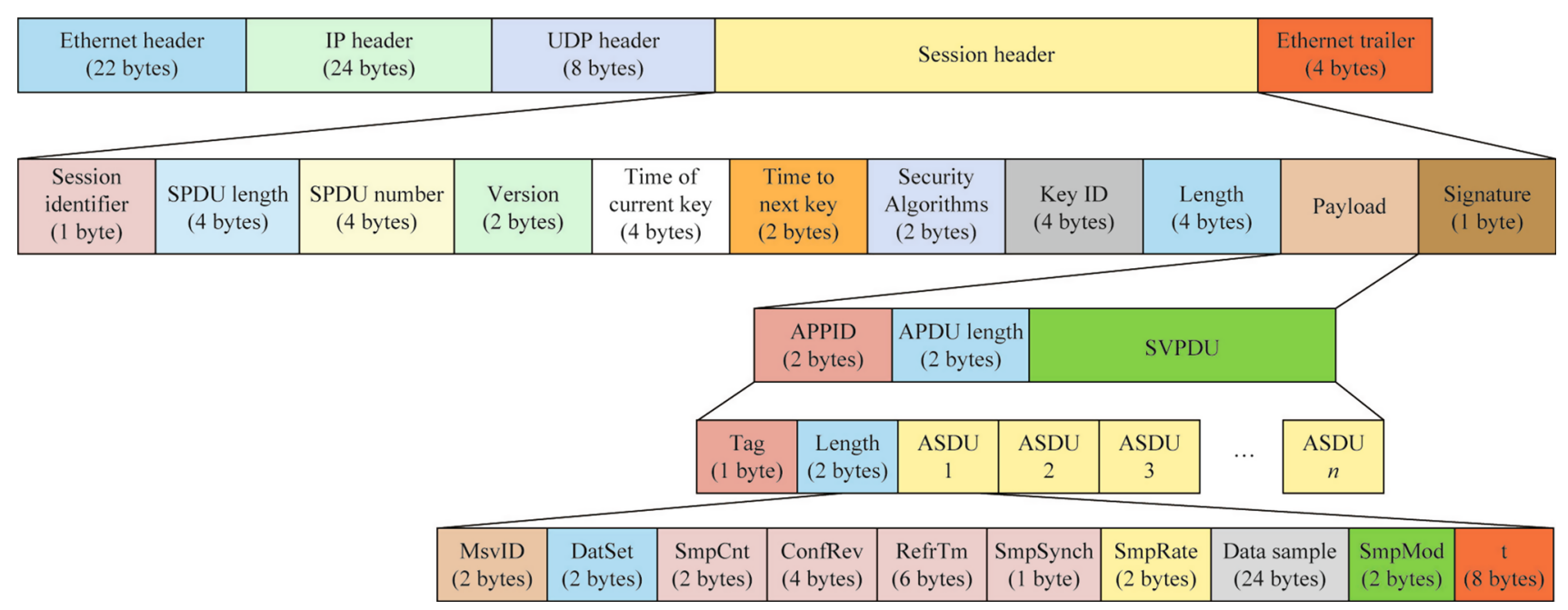

Fig. 6 Data word structure for IEC 61850-90-5 PMU 
subscribers. Generally publisher, i.e. PMU, is the sender of synchrophasor data and the subscriber, i.e. PDC, is its receiver. The key distribution center (KDC) provides the symmetric key coordination between the publishers and subscribers. Where a subscriber is given advanced warning of when the next key rotation is to occur, i.e. TimeToNextKey, and also detects if it is out-of-sync with the current key.

'Integrity' is a measure of accuracy of the data packets received. IEEE C37.118 includes cyclic redundancy check (CRC) code inside CHK bits of the packet to ensure integrity against any modification. The CRC code doesn't provide enough security mechanism and hence it may be accessed and modified by any intruder easily. Whereas in IEC 61850-90-5 uses digital signatures with asymmetric cryptography to provide information authentication and integrity. Another characteristic includes 'Availability' which ensures efficient communication between the publishers and subscribers and is left unaddressed in both IEEE C37.118.2 and IEC 61850-90-5 standards. Table 1 summarizes the comparison of some major features of IEEE C37.118.2 and IEC 61850-90-5 standards.

\section{Comparative performance evaluation of PMU communication networks}

In this paper, the performance of IEEE C37.118.2 and IEC 61850-90-5 based PMU communication network is tested using a modified IEEE 30-bus power system as shown in Fig. 7 [20]. Modification is done to include some distributed energy resources (DER) and circuit breakers at strategic locations so that it can be operated in three microgrids. The buses 1, 2, 3, 4, 5, 6, 7 can operate as an islanded microgrid with a generator at bus 1 and 2 supplying the necessary loads. The second islanded portion of the grid consists of buses $8,9,10,11,12,13,15,16,17,18$, 19, 20, 21, 22 and 28. This portion consists of four distributed energy resources (DERs) at buses 8, 11, 13 and 17.

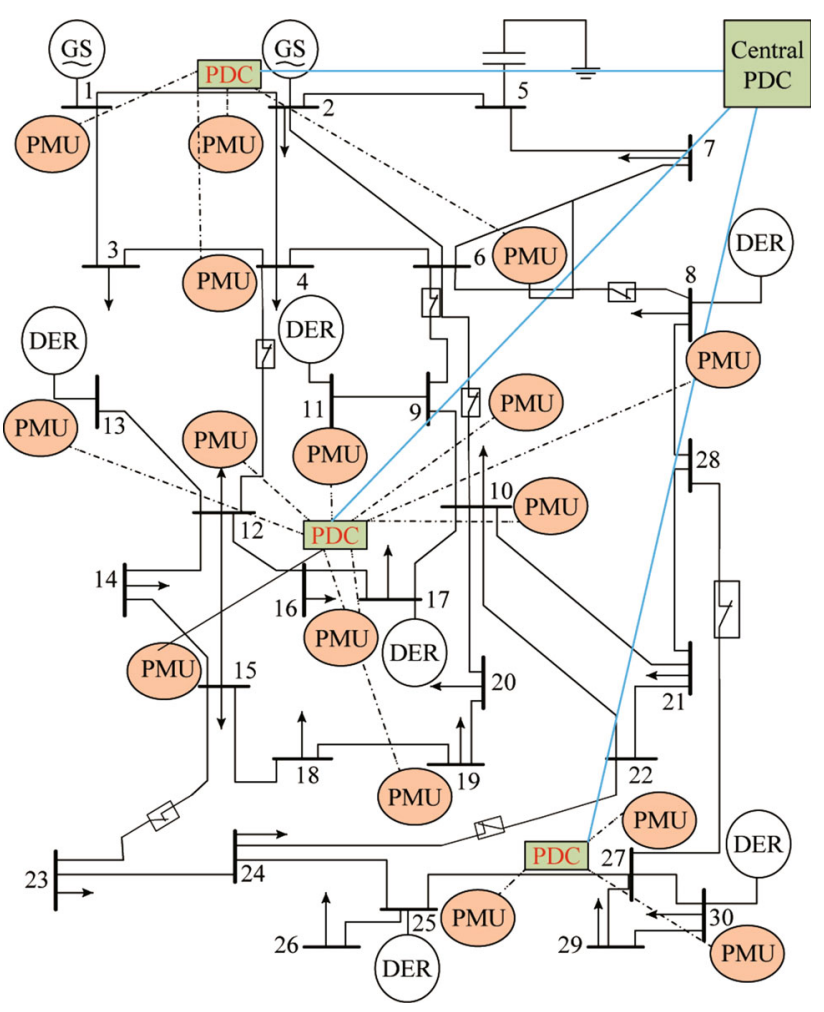

Fig. 7 Modified IEEE 30 bus system

The third islanded portion of the grid consists of buses namely, 23, 24, 25, 26, 27, 29 and 30. It consists of two DERs at buses 25 and 30 which are capable of supplying the load in the islanded portion independently.

In order to have optimum monitoring cost as well as optimum data aggregation at the control center or a PDC optimum PMU placement is resorted. The optimized PMU placement formulation is based on the fact that minimum number of PMU are to be placed in a network adhering to the condition that each bus must be reached at least once by a PMU. The problem formulation for integer linear programming (ILP) for an $\mathrm{N}$ bus power system can be done as in (1) subject to (2) [21].

Table 1 Comparison between IEEE C37.118.2 and IEC 61850-90-5 standards

\begin{tabular}{|c|c|c|}
\hline Features & IEEE C37.118.2 & IEC 61850-90-5 \\
\hline Protocol Stack & $\begin{array}{l}\text { For transport and network layers TCP or UDP over IP, } \\
\text { link and physical layer is as per user choice }\end{array}$ & $\begin{array}{l}\text { For transport and network layers UDP over IP, link } \\
\text { layer is Ethernet and physical layer is as per user } \\
\text { choice }\end{array}$ \\
\hline Sampling Rate & 10-30 samples/sec (for $50 \mathrm{~Hz}$ ) & 4000-12800 samples/sec (for $50 \mathrm{~Hz}$ ) \\
\hline Security features & Limited and more vulnerable to intrusion & $\begin{array}{l}\text { Provides a strong security mechanism by exchanging } \\
\text { cryptographic keys among devices. However } \\
\text { availability is still an issue }\end{array}$ \\
\hline Streaming protocol & Yes & Yes (R-SV) \\
\hline Average data word size & 112 bytes & 305 bytes \\
\hline
\end{tabular}


$\min \sum_{k=1}^{N} W_{k} X_{k}$

$\boldsymbol{A X} \geq \boldsymbol{b}$

where $W_{k}$ is a weighting factor, $X_{k}$ is a binary variable vector which contains 1 if a PMU is needed and 0 if PMU is not required, $\boldsymbol{A}$ is adjacency matrix whose elements $a_{i j}$ are defined as,

$a_{i j}= \begin{cases}1 & \text { if } i=j \\ 1 & \text { if } i \text { and } j \text { are connected } \\ 0 & \text { otherwise }\end{cases}$

and $\boldsymbol{b}$ is a vector which contains all ones and is defined as

$\boldsymbol{b}=\left[\begin{array}{lllll}1 & 1 & 1 & \ldots & 1\end{array}\right]^{\mathrm{T}}$

The locations for the optimal PMU placement for complete observability of the system according to the above formulated ILP are found to be at the buses 2, 4, 6, 9, $10,12,15,19,25$ and 27 of the test system. Moreover, in order to provide the out-of-step protection function for generators and DERs, PMUs must be placed at the generator \& DER buses [22] in addition to the above optimal placements. This approach makes phasor measurements, at generator \& DER buses, available with minimal estimation errors. In this case, the PMU placements for test system is found to be at buses $1,2,4$, $6,8,9,10,11,12,13,15,17,19,25,27$, and 30 , which includes generator \& DER buses and the buses obtained through ILP for optimal PMU placement.

The performance of the above PMU communication networks must meet the End-to-End (ETE) latency requirements for different applications listed out in Table 2 as specified by the standards for its successful and efficient operation.

In this paper, it is assumed that the test system is spread over an area of $1000 \mathrm{~km}$ by $1000 \mathrm{~km}$. Hence in optimized network engineering tool (OPNET) simulation [23], a project scenario with 1000 by $1000 \mathrm{~km}$ span area is set up. The 'ethernet_wkstn_adv' from the OPNET object palette is selected to model PMU, as it provides a communication

Table 2 ETE delays for different PMU wide area monitoring and control applications

\begin{tabular}{lll}
\hline Factor & Reporting rates & End to end latency \\
\hline Synchrocheck & $>4$ per s & $100 \mathrm{~ms}$ \\
Out of step protection & $>10$ per s & $50 \mathrm{~ms}$ \\
Adaptive relaying & $>10$ per s & $50 \mathrm{~ms}$ \\
State estimation & $1 / 300$ per & $5 \mathrm{~s}$ \\
Situational awareness & 1 per s & $5 \mathrm{~s}$ \\
Wide area control & $>10$ per s & $50 \mathrm{~ms}$ \\
\hline
\end{tabular}

structure with media access control (MAC) layer, IP layer, UDP layer, while 'ethernet_server_adv' node is selected to model regional and central PDCs. The PMUs are connected to different PDCs via switches and routers. Links of different data types for different scenarios are used for connecting the routers, e.g. "ATM OC3" links, which has a data rate of 155 Mbps. In order to simulate a WAN, each PMU at respective buses as shown in Fig. 7 are connected with routers and run in OPNET environment. The routers and switches have been appropriately chosen from the OPNET library.

In this communication network, two types of communication services have been invoked. First, the PMU measures the synchrophasor data at respective power system buses and transmits it to regional level PDC. Second, the regional level PDC combines the incoming synchrophasor messages from several PMUs and transmits them to central PDC.

The PMU communication networks based on both IEEE C37.118.2 and IEC 61850-90-5 are simulated as two different scenarios in OPNET modeler. Also each scenario has been evaluated for dedicated and shared networks for different link types. In order to evaluate performance over a shared communication network, a constant background traffic has been simulated in the network. This background traffic has been modelled as a stochastic data flow. As the traffic in the networks is normally fractal and self-similar in nature [24], Pareto distribution is selected as the probability distribution function to model background traffic flows [25]. The background traffic in OPNET modeler is set using the "background traffic config" node, under its attributes the packet interarrival time is set as Pareto distribution. The data packets suffer larger delays in presence of background traffic over the network.

In OPNET modeler, the node "Application Config" is used to model the different traffic flows and its corresponding attributes such as inter-arrival time, size of each packet etc. In IEEE C37.118.2 based PMU communication networks, three applications corresponding to command frame, configuration frame and data frames are created in "Application Config" node. At the beginning of simulation, the command and configuration frames are set, followed by data frames. The file size of data frame to be transferred is selected as 112 bytes as discussed in Sect. 2 . Whereas for IEC 61850-90-5 PMU communication networks, three applications namely MMS request-response, R-GOOSE and R-SV are created in "Application Config" node. The MMS request-response messages are set to be exchanged at the start of the simulation and its size is set to 215 bytes. The R-GOOSE application is configured with a R-GOOSE message which has "SvEna" value of R-MSCVB control block set to 1 , having a size of 142 bytes. The R-SV application is configured with R-SV 
Table 3 Average ETE time delay of IEEE C37.188.2 and IEC 61850-90-5 based PMU communication networks under different links for modified IEEE 30 bus system

\begin{tabular}{|c|c|c|c|c|c|}
\hline \multirow[t]{3}{*}{ Type of WAN link } & & \multicolumn{4}{|c|}{ Average ETE time delay in PMU communication network (ms) } \\
\hline & & \multicolumn{2}{|l|}{ IEEE C37.118.2 } & \multicolumn{2}{|l|}{ IEC 61850-90-5 } \\
\hline & & $\begin{array}{l}\text { No background } \\
\text { traffic }\end{array}$ & $\begin{array}{l}\text { With background } \\
\text { traffic }\end{array}$ & $\begin{array}{l}\text { No background } \\
\text { traffic }\end{array}$ & $\begin{array}{l}\text { With background } \\
\text { traffic }\end{array}$ \\
\hline \multirow[t]{2}{*}{ ATM_OC1 (51.84 Mbps) } & PMU to regional PDC & 8.6 & 13.0 & 15.2 & 22.2 \\
\hline & Regional PDC to Central PDC & 14.0 & 32.0 & 39.0 & 46.0 \\
\hline \multirow[t]{2}{*}{ ATM_OC3 (155.52 Mbps) } & PMU to regional PDC & 2.1 & 3.0 & 6.3 & 8.3 \\
\hline & Regional PDC to Central PDC & 6.0 & 8.0 & 12.0 & 15.0 \\
\hline \multirow[t]{2}{*}{ ATM_OC12 (622.08 Mbps) } & PMU to regional PDC & 2.0 & 2.2 & 4.5 & 5.1 \\
\hline & Regional PDC to Central PDC & 4.2 & 4.5 & 8.5 & 9.2 \\
\hline
\end{tabular}

frames of 305 bytes, as discussed in Sect. 3. The transmission of R-SV messages is terminated at the end of simulation by sending R-GOOSE message with "SVEna" value of R-MSCVB set to 0 . By combining different application over a period of time, different profiles are created, using "profile config" node, to set traffic flows between different PMUs and PDCs. Two different profiles, "PMU_to_rPDC" and "rPDC_to_cPDC' for traffic configurations between PMU to regional PDCs and regional PDCs to central PDCs are created. After setting the traffic in scenarios, the simulation is run for $10 \mathrm{~min}$.

From Table 3, it is evident that when background traffic is available in the network, the ETE delay for messages from PMU to regional PDC and regional PDC to central PDC is increased. The ETE delay for message delivery from regional PDC to central PDC is greater as compared to the ETE delay from PMU to regional PDC due to aggregation of data from different PMUs. From Table 3, it can be seen that the ETE delay for messages from PMU to regional PDC for different links of WAN in IEEE C37.118.2 based PMU communication network is less than IEC 61850-90-5 based PMU communication networks. This is due to the fact that the sampling rate of IEC 61850-90-5 based PMU messages is very high and the size of data message is almost three times that of IEEE C37.118.2 data message. Also, the high sampling rates of IEC 61850-90-5 based PMUs pave way for utilizing the PMU data for many protection, control and monitoring functions where high sampling rates are required. The ETE delay for IEC 61850-90-5 PMU messages is still well below the specified requirements for different applications as given in Table 2 .

The ETE delay for IEC 61850-90-5 PMU communication networks is within the specified limits even for the "ATM_OC_1" type links which has a data rate of 51.84 Mbps. It is evident from Table 3 that for links with higher bandwidths, the ETE delay in the networks is decreasing. Proposed idea for design, modeling and performance testing of IEC 61850-90-5 PMU communication networks is equally valid for any size of PMU communication networks, since change in network size only changes the traffic scenario which is addressed by choosing appropriate network/link bandwidths. Also, IEC 61850-90-5 PMU communication networks have more secure, scalable, interoperable communication framework when compared to IEEE C37.118.2 based PMU communication networks.

\section{Conclusion}

A new information model and its services are presented in this paper for PMU. This PMU model is based on IEC 61850-90-5. This PMU model has used some of the already existing logical nodes related to SVs and GOOSE and added few new data objects to these logical nodes. This effort has helped in extending the tested services of IEC 61850, i.e. SVs and GOOSE, on a PMU WAN in the form of R-SV and $\mathrm{R}-\mathrm{GOOSE}$. This is done to provide an insight for communication needs along with an interoperable PMU model based on IEC 61850. Various messages/services exchange for existing IEEE C37.118.2 and new IEC 61850-90-5 PMU communication networks are analyzed and compared. This comparison has provided a way for the development of an IEC 61850 compliant PMU and its easy substitution for the existing IEEE C37.118.2 based PMU communication. The evaluation of the underlying PMU communication networks are in terms of ETE delay for different traffic load scenarios and under different types of links. The ETE delay results are found to be in conformance with the benchmark set by the standards for monitoring, protection and control applications. This study demonstrates the suitability of IEC 61850-90-5 based PMUs model for IEC 61850 utility automation systems. Also, IEC 61850-90-5 PMU communication networks are found to be more secure, scalable and interoperable as compared to IEEE C37.118.2 based PMU communication networks. 
Open Access This article is distributed under the terms of the Creative Commons Attribution 4.0 International License (http:// creativecommons.org/licenses/by/4.0/), which permits unrestricted use, distribution, and reproduction in any medium, provided you give appropriate credit to the original author(s) and the source, provide a link to the Creative Commons license, and indicate if changes were made.

\section{References}

[1] Phadke AG (1993) Synchronized phasor measurements in power system. IEEE Comput Appl Power 6(2):10-15

[2] Chakrabarti S, Kyriakides E (2008) Optimal placement of phasor measurement units for power system observability. IEEE Trans Power Syst 23(3):1433-1440

[3] Huang L, Sun Y, Xu J et al (2014) Optimal PMU placement considering controlled islanding of power system. IEEE Trans Power Syst 29(2):742-755

[4] Yang P, Tan Z, Wiesel A et al (2015) Placement of PMUs considering measurement phase angle mismatch. IEEE Trans Power Deliv 30(2):914-922

[5] Xia N, Gooi HB, Chen SX et al (2015) Redundancy based PMU placement in state estimation. Sustain Energy Grid Net 2:23-31

[6] Kamwa I, Pradhan AK, Joos G (2011) Adaptive phasor and frequency-tracking schemes for wide-area protection and control. IEEE Trans Power Deliv 26(2):744-753

[7] Yan J, Liu CC, Vaidya U (2011) PMU-based monitoring of rotor angle dynamics. IEEE Trans Power Syst 26(4):2125-2133

[8] IEEE Std 1344-2001. IEEE standard for synchrophasor for power systems (2001)

[9] IEEE Std C37.118 - 2005. IEEE standard for synchrophasors for power systems (2005)

[10] IEEE C37.118.1-2011. IEEE standard for synchrophasor measurements for power systems (2011)

[11] IEEE C37.118.1a-2014. IEEE standard for synchrophasor measurements for power systems-Amendment 1: modification of selected performance requirements (2014)

[12] IEEE C37.118.2-2011. IEEE standard for synchrophasor data transfer for power systems (2011)

[13] Chenine M, Nordstrom L (2011) Modeling and simulation of wide-area communication for centralized PMU-based applications. IEEE Trans Power Deliv 26(3):1372-1380

[14] Naduvathuparambil B, Valenti MC, Feliachi A (2002) Communication delays in wide area measurement systems. In: Proceedings of the 34th southeastern symposium on system theory, Huntsville, AL, USA, 18-19 Mar 2002, pp 118-122

[15] Zhang F, Sun Y, Cheng L et al (2015) Measurement and modeling of delays in wide-area closed-loop control systems. IEEE Trans Power Syst 30(5):2426-2433

[16] IEC 61850 (2013) Communication networks and systems for power utility automation

[17] IEC 61850-90-5: 2012. Communication networks and systems for power utility automation-Part 90-5: use of IEC 61850 to transmit synchrophasor information according to IEEE C37.118 (2012)
[18] Abdolkhalig A, Zivanovic R (2014) Phasor measurement based on IEC 61850-9-2 and Kalman-filtering. Measurement 50(1):126-134

[19] Lee JD, Lee SJ, Bae JH, et al (2013) The PMU interface using IEC 61850. In: Proceedings of the 2013 international conference on ICT convergence (ICTC'13), Jeju, Republic of Korea, 14-16 Oct 2013, pp 1125-1128

[20] Power systems test cases archive. Electrical Engineering, University of Washington, St Louis, MO, USA (1999)

[21] Gou B (2008) Generalized integer linear programming formulation for optimal PMU placement. IEEE Trans Power Syst 23(3):1099-1104

[22] Lin H, Sambamoorthy S, Shukla S, et al (2012) A study of communication and power system infrastructure interdependence on PMU-based wide area monitoring and protection. In: Proceedings of the 2012 IEEE power and energy society general meeting, San Diego, CA, USA, 22-26 July 2012, 7 pp

[23] OPNET modeler (riverbed modeler). Riverbed Technology Inc, San Francisco, CA, USA

[24] Leland WE, Taqqu MS, Willinger W et al (1994) On the selfsimilar nature of ethernet traffic (extended version). IEEE/ACM Trans Netw 2(1):1-15

[25] Zhang W, He J (2007) Modeling end-to-end delay using Pareto distribution. In: Proceedings of the 2 nd international conference on internet monitoring and protection (ICIMP'07), San Jose, CA, USA, 1-5 July 2007, 21 pp

Ikbal ALI graduated from Aligarh Muslim University, Aligarh, received the M.Tech. degree from the Indian Institute of Technology, Roorkee, India and has a Ph.D. in Electrical Engineering. Presently, he is Associate Professor in the Department of Electrical Engineering, Jamia Millia Islamia (A Central University), New Delhi. As principle investigator, Dr. Ali is executing research projects on Substation Automation, Micro-grid and IEC 61850 based utility automation funded from DST, AICTE, JMI and IEEE Standards Education Society. His research interests include IEC 61850 based utility automation, substation communication networks architecture and smart grid.

Mohd Asim AFTAB received B.Tech. (Hons.) degree in Electrical \& Electronics Engineering from Uttar Pradesh Technical University, Lucknow, in 2012. He received M.Tech. degree in Control \& Instrumentation Systems in 2015 and currently pursuing the Ph.D. degree in Electrical Engineering both from Jamia Millia Islamia (A Central University), New Delhi, India. His research interests include active distribution systems, power system communications, wide area control and measurement systems.

S. M. Suhail HUSSAIN received B.Tech. degree in Electrical \& Electronics Engineering from Sri Venkateswara University, Tirupati, India, in 2010 and the M.Tech. degree from Jawaharlal Nehru Technological University, Anantapur, India in 2013. He is currently pursuing the Ph.D. degree in Electrical Engineering at Jamia Millia Islamia (A Central University), New Delhi, India. His research interests include microgrid, power system communications and smart grid. 